\title{
Featuring the phenotype of the FMF prototype
}

\author{
I Ben-Zvi , Y Kassel, O Kukuy, C Herskovizh, C Grossman, A Livneh \\ From 8th International Congress of Familial Mediterranean Fever and Systemic Autoinflammatory Diseases \\ Dresden, Germany. 30 September - 3 October 2015
}

\section{Background}

The presentation of FMF is extremely variable, ranging from a quiescent to severe disabling disease. The M694V mutation is one of approximately 300 published genetic variations of MEFV and is thought to be associated with a typical clinical picture of the disease, but studies featuring the phenotype of homozygous M694V phenotype are meager.

\section{Objectives}

To describe the clinical trait of M694V homozygous FMF as compared to the phenotype of FMF with mixed MEFV genotypes.

\section{Patients and methods}

Fifty seven FMF patients homozygous for the M694V genotype were compared to 56 patients carrying other mutations. A questionnaire, including items related to demographic and clinical features was completed for each patient based on interview, physical examination and file notes.

\section{Results}

Compared with the control group, more patients, homozygous for the M694 mutation, suffered from a severe disease ( $\mathrm{p}=0.001)$, had higher frequency of attacks before and during colchicine treatment $(\mathrm{p}=0.0001$ and 0.0007 , respectively), had more related diseases $(\mathrm{p}=0.0373)$ and needed higher dose of colchicine to control their disease $(\mathrm{p}=0.0001)$. Most other features tested (Table 1) appeared to be more pronounced in M694V homozygous patients (either with or without statistical significance).

\section{Conclusion}

The phenotype of FMF, as manifested in M694V homozygous patients, is the gold standard, to which other FMF presentations should be compared.

Published: 28 September 2015
Table 1

\begin{tabular}{llll}
\hline Parameter & $\begin{array}{l}694 \\
\text { Homozygous } \\
(\mathrm{N}=57)\end{array}$ & $\begin{array}{l}\text { Other } \\
\text { mutations } \\
(\mathrm{N}=56)\end{array}$ & $\mathrm{P}$ \\
\hline $\begin{array}{l}\text { Average length of attack } \\
\text { (days) }\end{array}$ & $2.66 \pm 1.5$ & $3.03 \pm 1.2$ & 0.073 \\
\hline Abdominal attacks & $50(87.7 \%)$ & $48(85.7 \%)$ & 0.788 \\
\hline Arthritis attacks & $52(91.3 \%)$ & $28(50 \%)$ & $<\mathbf{0 . 0 0 0 1}$ \\
\hline Pleuritis attacks & $36(46.2 \%)$ & $18(38.2 \%)$ & $\mathbf{0 . 0 0 1 3}$ \\
\hline Exertional leg-pain & $47(82.5 \%)$ & $36(64.3 \%)$ & $\mathbf{0 . 0 3 4}$ \\
\hline ELE attacks & $10(17.5 \%)$ & $3(5.4 \%)$ & 0.073 \\
\hline Attacks of fever alone & $20(35.1 \%)$ & $12(21.4 \%)$ & 0.143 \\
\hline $\begin{array}{l}\text { Average colchicine dose } \\
\text { (mg/day) }\end{array}$ & $1.9 \pm 0.48$ & $1.48 \pm 0.54$ & $\mathbf{0 . 0 0 0 1}$ \\
\hline IV colchicine treatment & $5(8.8 \%)$ & $0(0 \%)$ & 0.057 \\
\hline Proteinuria or amyloidosis & $6(10.5 \%)$ & $1(1.8 \%)$ & 0.113 \\
\hline Anemia of chronic disease & $14 / 53(26.4 \%)$ & $7 / 52(13.5 \%)$ & 0.142 \\
\hline $\begin{array}{l}\text { Elevated acute phase } \\
\text { reactants }\end{array}$ & $10 / 18(55.6 \%)$ & $4 / 16(25 \%)$ & 0.092 \\
\hline Chronic renal failure & $6(10.5 \%)$ & $0(0 \%)$ & $\mathbf{0 . 0 2 7}$ \\
\hline Chronic arthritis & $11(19.3 \%)$ & $2(3.6 \%)$ & $\mathbf{0 . 0 1 5}$ \\
\hline $\begin{array}{l}\text { Work days lost each } \\
\text { month }\end{array}$ & $4.4 \pm 7.2$ & $2.6 \pm 4.6$ & 0.718 \\
\hline Harm to quality of life & $(1-10) 5.6 \pm 3.3$ & $4.1 \pm 3$ & $\mathbf{0 . 0 1 3}$ \\
\hline $\begin{array}{l}\text { Number of attacks per year } \\
\text { W colchicine }\end{array}$ & $7.2 \pm 7.8$ & $3.5 \pm 5.5$ & $\mathbf{0 . 0 0 0 7}$ \\
\hline $\begin{array}{l}\text { Number of attacks per year } \\
\text { w/o colchicine }\end{array}$ & $23.6 \pm 9.3$ & $15.6 \pm 11.7$ & $\mathbf{0 . 0 0 0 1}$ \\
\hline Crohn's disease & $4(7 \%)$ & $2(3.6 \%)$ & 0.679 \\
\hline Ankylosing Spondylitis & $3(5.3 \%)$ & $1(1.8 \%)$ & 0.619 \\
\hline Behcet's Disease & $7(12.3 \%)$ & $1(1.8 \%)$ & 0.061 \\
\hline Henoch Schonlein Purpura & $1(1.8 \%)$ & 0 & 1 \\
\hline All FMF associated diseases & $17(29.8 \%)$ & $7(12.5 \%)$ & $\mathbf{0 . 0 3 7 3}$ \\
\hline
\end{tabular}

doi:10.1186/1546-0096-13-S1-P78

Cite this article as: Ben-Zvi et al:: Featuring the phenotype of the FMF

prototype. Pediatric Rheumatology 2015 13(Suppl 1):P78. 